\title{
Artificial Intelligence Hybrid System for Enhancing Retinal Diseases Classification Using Automated Deep Features Extracted from OCT Images
}

\author{
Amin Alqudah $^{1}$, Ali Mohammad Alqudah ${ }^{* 2}$, Ma'moun AlTantawi ${ }^{3}$
}

\section{Submitted: 16/04/2021 Accepted : 22/08/2021}

\begin{abstract}
After the advent of eye 2D imaging technology, Optical Coherence Tomography (OCT) has become one of the most effective and commonly used imaging techniques for non-invasive retinal eye disease evaluation. Blindness is primarily diagnosed using OCT with one of the following two eye diseases categories: diabetic macular edema (DME) or age-related macular degeneration (AMD). The classification of eye retina diseases using OCT images has recently become a challenge with the development of machine learning and profound learning techniques. In this paper, a hybrid artificial intelligence system for multiclass classification of eye retina diseases has been proposed, using automated deep features extracted, based on Advanced OCT Network (AOCTNet) architecture from OCT images especially spectral-domain (SD-OCT) images. The proposed methodology mainly can be used to classify retinal diseases into normal and four abnormal classes (AMD, choroidal neovascularization (CNV), DME, and Drusen). The proposed system was constructed using eight types of machine learning algorithms (Support Vector Machine with Linear kernel (LSVM), Support Vector Machine with Radial Basis Function kernel (RBF SVM), Artificial Neural Network (ANN), K Nearest Neighbor (KNN), Random Forest (RF), Linear Discriminant Analysis (LDA), Quadratic Discriminant Analysis (QDA), and Naïve Bayes (NB)). The classifiers achieved high performance. For example, the KNN and the RF classifiers achieved an accuracy of 99.44 and 99.12 , respectively. This methodology is potentially a powerful computer-aided diagnostic (CAD) tool for the use of SD-OCT imaging for retinal diseases.
\end{abstract}

Keywords: OCT, AOCTNet, Deep Learning, Machine Learning, Feature Extraction, Hybrid Systems

This is an open access article under the CC BY-SA 4.0 license. (https://creativecommons.org/licenses/by-sa/4.0/)

\section{Introduction}

A film of the optic nerve and light-sensitive tissue surrounding the inner surface of the eyeball is found in the retina inside the human eye. The concentrated light is received by the eye lens and transformed into neural signals [1]. The Macula layer is the largest sensing area in the central part of the retina which contains different layers of nerve photoreceptor cells responsible for colour recognition. Eventually, the retina processes the data obtained from the macula and sends it to the brain for visual recognition through the optic nerve [1], [2].

Some eye disorders that can influence the retina are among the main triggers of vision impairment in western populations, with more than 300 million DME cases which means that DME may become a rising issue in the next few years [3],[4],[5]. Examples of that: Age-related macular degeneration (AMD), choroidal neovascularization $(\mathrm{CNV})$, drusen, and diabetic macular edema (DME). Such disorders could lead to blindness and impact lifestyles. Retina diseases are the most crucial diseases that affect human eyes, based on that, scientists are motivated to explore new and effective tools for diagnosing eye conditions [6],[7].

An optical coherence tomography (OCT), with approximately 5.35

\footnotetext{
${ }^{1}$ Department of Computer Engineering, Yarmouk University, Irbid, Jordan. ORCID ID: 0000-0002-2538-3402

${ }^{2}$ Department of Biomedical Systems and Informatics Engineering, Yarmouk University, Irbid, Jordan. ORCID ID: 0000-0002-5417-0043 ${ }^{3}$ Department of Electronics Engineering, Yarmouk University, Irbid, Jordan. ORCID ID: 0000-0003-4961-8226

*Corresponding Author Email: ali_qudah@hotmail.com
}

million OCT scans conducted in 2014 alone in the United States is one of the most popular imaging modalities for diagnosing the eye [8]. OCT is a non-invasive imaging tool useful for diagnosing eye retina disease [8]. OCT imaging allows ophthalmologists to see distinctive retinal layers, to calculate, monitor, and direct the treatment [9], [10]. OCT can capture and generate images in two different domains: the Time domain and spectral domain. OCT usually results in significant changes in imagery efficiency; offering more detailed knowledge regarding intraretinal retaining layer morphology that is used for the identification and diagnosis of diseases [11].

Nowadays, many researchers are focusing on applying OCT on medical data to improve medical decisions [12],[13],[14], [36], [37], and [38]. This is done by applying and improving artificial intelligence (AI) in particular, machine learning (ML), and deep learning (DL) algorithms, by focusing on using these algorithms separately or together. Many researchers have worked on this area (diagnosis systems) such as the proposed work in [15],[16],[17]. The key necessity for this work is having a huge OCT retina dataset either in time or spectral domains as suggested by [1] or by [2].

Deep learning (DL) is the state-of-the-art Artificial Intelligence (AI) branch used to apply multi-layer computation models and understand how data can be interpreted in various abstraction layers [12],[13],[14]. Convolutional Neural Network (CNN) technique is a state-of-the-art DL approach used for the diagnosis of medical diseases, especially image-based diseases [13]. CNN is structured in general to reduce the pre-processing of data and to 
view raw images. There are several layers in the $\mathrm{CNN}$ : input layer, convolution layer, RELU layer, classification layer, and output layer [1]. CNN is primarily based on two stages; the first one is the convolution stage, and the second one is downsampling. The convolution stage is carried out with pre-specified size and weight training filters [1], [13]. The downsampling stage is responsible for reducing the number of output data. The application of CNN is carried out in two approaches; the first approach is more widely used and is focused on the transfer of pretrained CNN templates and the grouping of new categories rather than their initial categories [16]. The second approach is to develop a particular
CNN for a specific use [15].

This study proposes a hybrid system of deep learning and machine learning approaches. Deep learning will be used as an automated deep feature extractor, while machine learning will be used as a classifier for enhancing automated multi-class detection based on raw SD-OCT images. The system is based on AOCTNet CNN to extract features from five types of retinal conditions (four disorder cases and normal cases). Figure 1 indicates the block diagram of the proposed methodology.

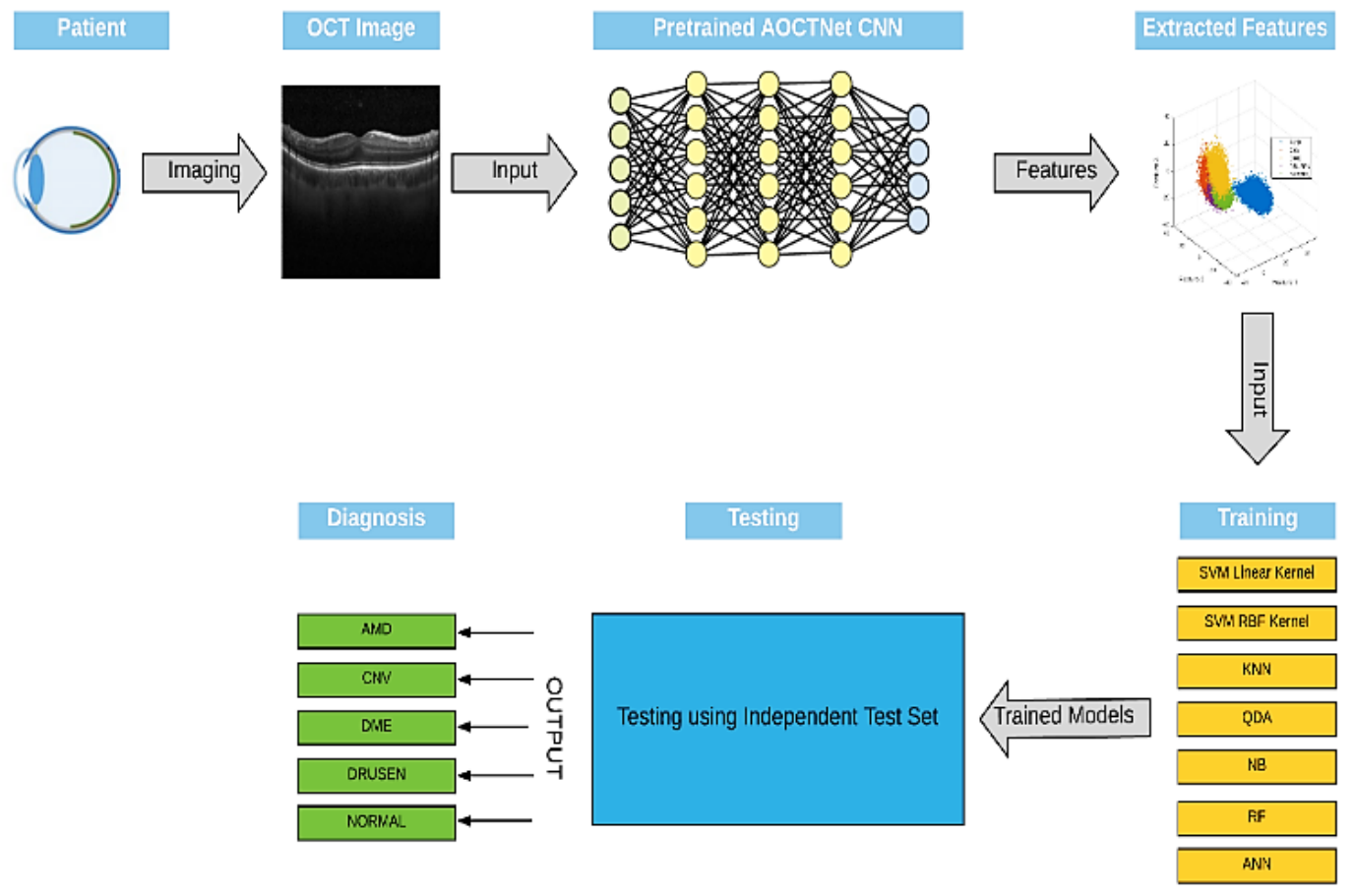

Fig. 1. Approach to Developing a Hybrid System for Eye Diagnosing using SD-OCT.

The contributions of this work can be summarized as follows:

- A new methodology using automated deep features extraction is proposed and trained.

- Enhancing the Classification of five classes of retina diseases.

- Using a huge dataset of SD-OCT images collected from different sources in the training and testing stages.

This paper is structured to include: Section 2 provides details about recent related works. Section 3 presents -with a detailed explanation- the used dataset and the proposed architecture. Section 4 discusses the results, including the performance of the proposed system. Section 5 is the discussion about the method results. And finally, section 6 presents the conclusion of the work.

\section{Literature Review}

Machine learning and deep learning approaches are more commonly used to recognize and diagnose retinal eye disorders. The most recent associated research articles are covered in this section. A system of unsupervised extraction of the features of the anterior chamber OCT pictures for ordering and classification purposes was proposed by Amil et al. [18]. The unsupervised approach consists of three key steps; pre-process images, calculating the gap between each pair of the images, and implementing a machine learning algorithm that uses the calculated distance to organize and identify items. The approach is implemented with a dataset consisting of 1000 OCT images.

Ji et al. [19] developed a system based on transfer learning using the CNN Inception V3 pre-trained framework. The method is used to classify the OCT images into two classes of diseases (dry AMD and DME) in addition to Normal cases of retinal OCT images. The total system accuracy was around $98 \%$ using the Beijing clinical datasets. While Perdomo et al. [20] designed a CNN called OCTNET which is made up of 12 layers. The designed CNN was used to characterize DME using SD-OCT quantities. The proposed CNN was evaluated by the Singapore Eye Research Institute (SERI) database covering 32 SD-OCT volumes. The overall accuracy was $93.75 \pm 3.125 \%$.

Nugroho [21] proposed a system that performs a comparison between two methods of extracting features: handcrafted and deep learning. This system aims to choose the best features and models that can be used to distinguish SD-OCT images. Handcrafted features include Local Binary Pattern (LBP), and Oriented Gradient Histogram (HOG). Deep learning features were extracted using DenseNet-169 and ResNet50 CNN models. The outcome reveals that deep learning features-based systems outperformed handcrafted features-based systems, with an accuracy of $88 \%$ and $89 \%$ for DenseNet and ResNet50, respectively. HOG and LBP achieved an accuracy of just $50 \%$ and $42 \%$, respectively.

Hwang et al. [22] developed a cloud-based artificial intelligence 


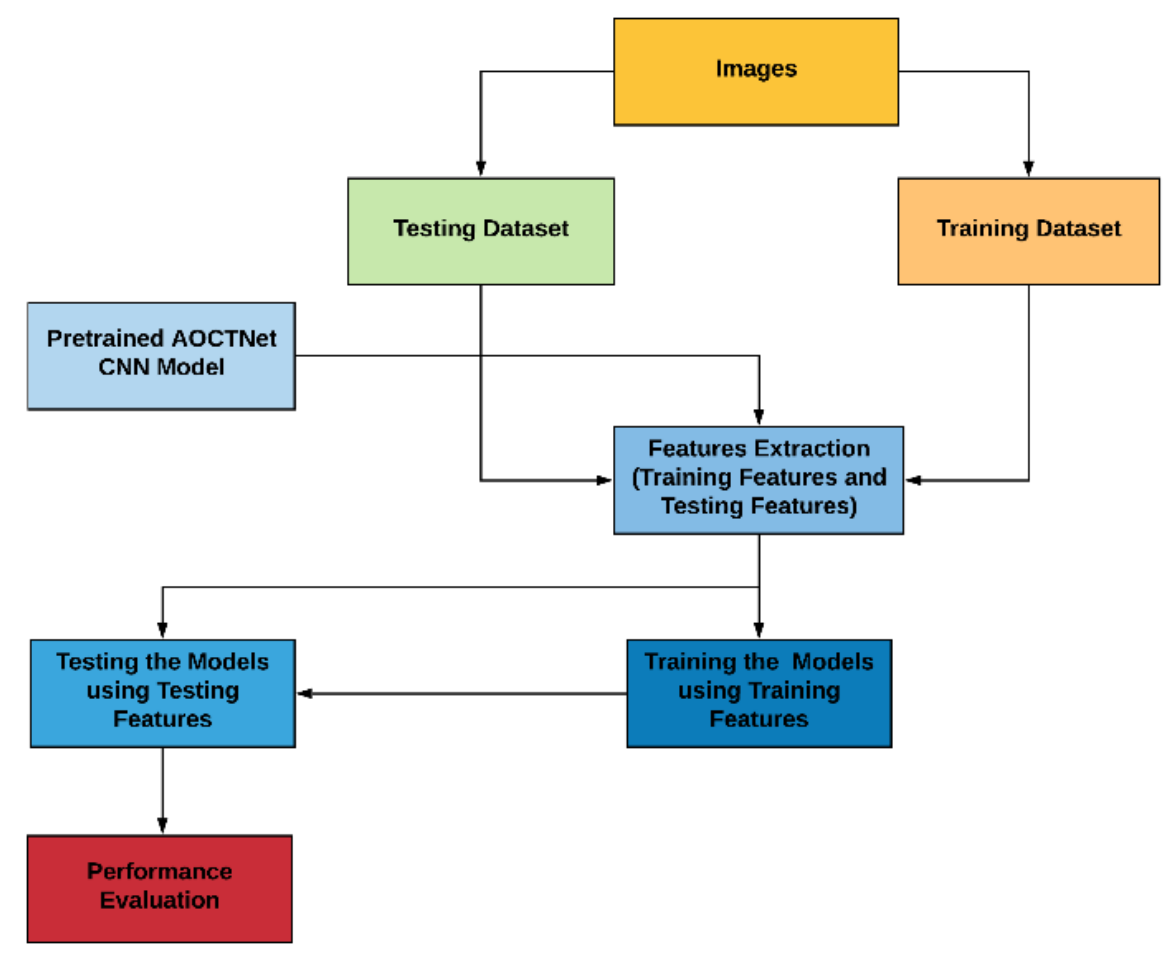

Fig. 2. Representative Hybrid System Workflow Diagram.

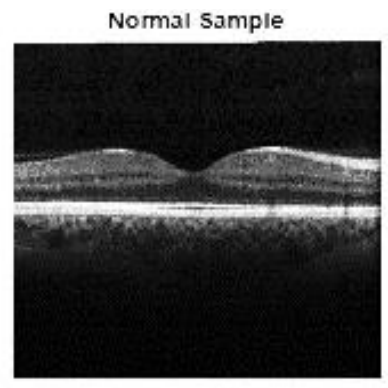

a)

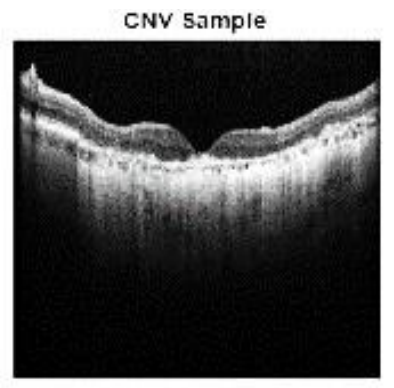

b)

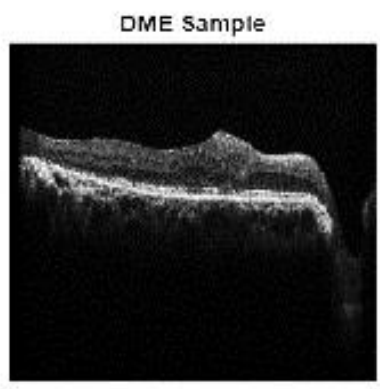

c)

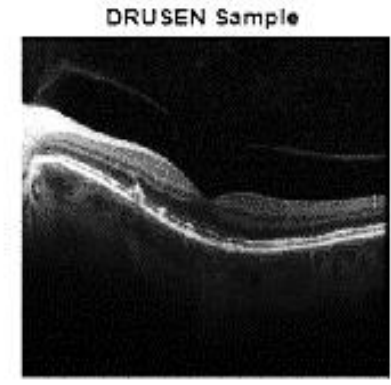

d)

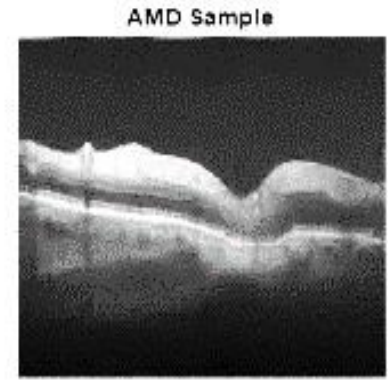

e)

Fig. 3. Samples of the images in the system for five classes: a) Normal, b) CNV, c) DME, d) DRUSEN, e) AMD

telemedicine web-based decision-making platform for diagnosis and treatment of AMD cases. The authors claimed that they used 35,900 labeled OCT images from AMD patients to train three separate pre-trained CNN models to build the systems. The pretrained CNN models were VGG16, InceptionV3, and ResNet50. The findings indicate that the used models obtained an accuracy of $91.20 \%, 96.93 \%$, and $95.87 \%$ for VGG16, InceptionV3, and
ResNet50, respectively. The precision and the responsiveness scores were no less than $90 \%$. Alqudah [1] proposed a CNN design called Advanced OCT network (AOCTNet) that is made up of 19 layers. The designed CNN model is used to classify the SD-OCT images into five classes (AMD, CNV, DME, Druses, and Normal) which makes this CNN model very efficient. The designed CNN model was trained, validated, and tested on the largest dataset in 


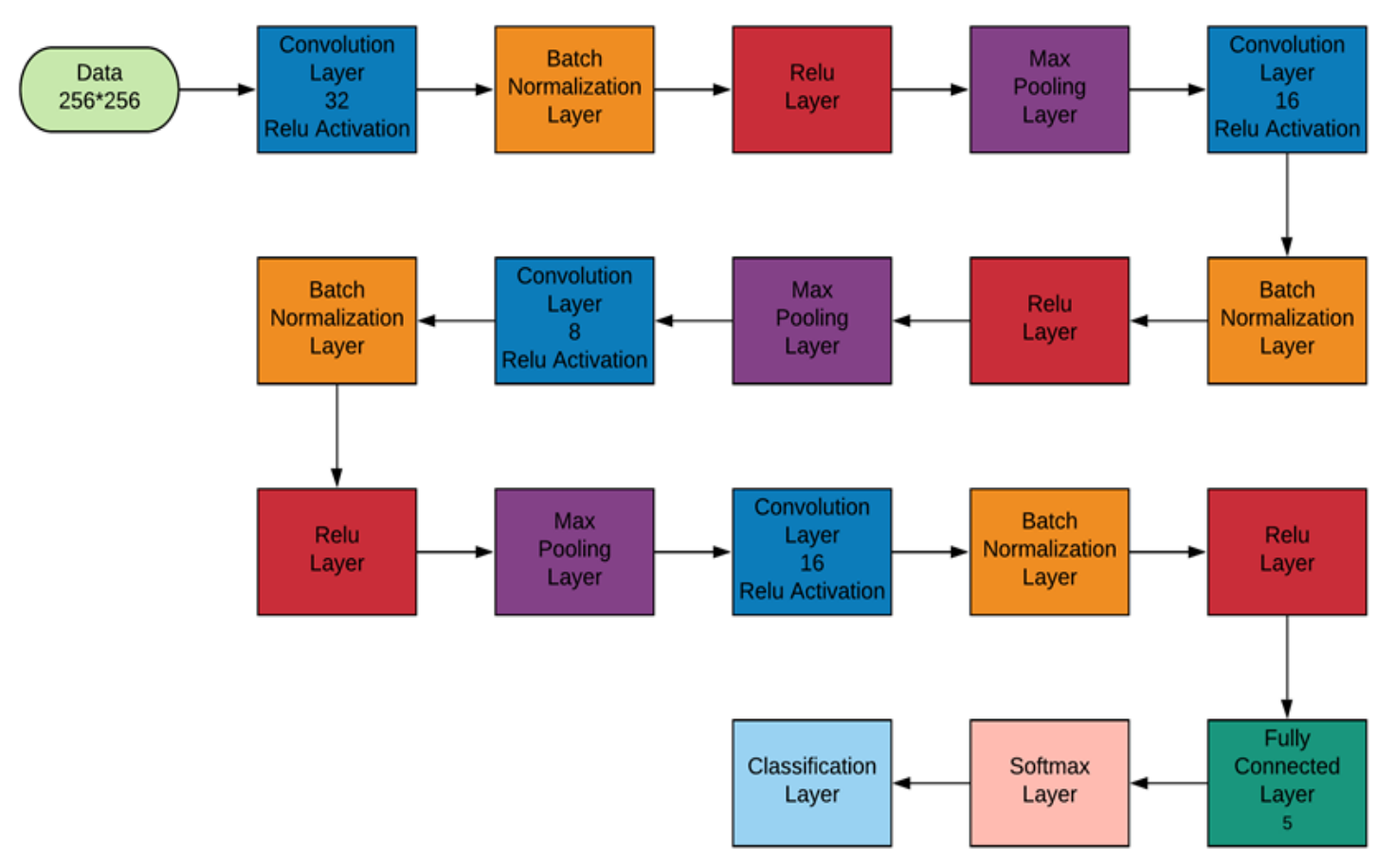

Fig. 4. The AOCTNet $\mathrm{CNN}$ architecture.

this literature with 137,437 SD-OCT images and achieved an accuracy of $97.1 \%$.

In this paper, a hybrid artificial intelligence system based on deep features extraction is used to classify the SD-OCT images into five classes. The proposed system fed the extracted deep features using AOCTNet to eight different classifiers to enhance classification performance.

\section{Materials and Methods}

The proposed methodology is addressed in this section. This section will cover the used datasets, the used AOCTNet CNN design, the extracted features, the used classifiers, and the performance evaluation techniques. Figure 2 displays the flow map of the suggested technique. Dataset

In this paper, we used the dataset that has been used in our research [1]. The used dataset consists of two huge datasets; the first one is the OCT dataset published by Zhang Lab at the University of California at San Diego (UCSD) [2]. While the second dataset is Farsiu 2013 Ophthalmology AMD dataset available at Duke University [3, 23].

The fusion of both datasets results in a dataset consists of 136,187 SD-OCT images with the following distribution: 51390 normal images, 37455 images with choroidal neovascularization, 11576 images with diabetic macular edema, 8866 images with drusen, and 26900 images for the age-related macular degeneration. For testing purposes, a dataset of 1,250 images ( 250 for each case) is collected from five different hospitals and private clinics [1]. The images are resized to a dimension of $256 \times 256$ to make them compatible with the input size of the used AOCTNet CNN architecture. Figure 3 shows samples of the used images.

\subsection{AOCTNet}

In general, $\mathrm{CNN}$ is being used for three main purposes: classification, feature extraction, and segmentation. It mainly consists of two main steps: the feature extraction step and the classification step; with a group of layers in each step. In the feature extraction step, each layer takes its input immediately from the previous layer output, then, it passes the output as an input to the next layer; while in the classification step, the layers receive their feature vectors as an input coming from the previous fully connected layer [24]. In this paper, we have used the AOCTNet CNN architecture that was proposed by Alqudah [1] for deep feature extraction. The AOCTNet consists of 19 layers to classify the SD-OCT images effectively. The AOCTNet has been trained on a huge number of SD-OCT images from different resources. The authors reported that the AOCTNet was trained using more than 137,000 SD-OCT images; making it suitable for deep feature extraction. Figure 4 shows the architecture of AOCTNet.

\subsection{Automated Deep Features Extraction}

The pre-trained CNN (AOCTNet) used in this study was used to extract deep features automatically from the SD-OCT images dataset. In the current research, the fully connected layer of the used $\mathrm{CNN}$ was used as a feature extraction layer; this layer precedes the classification layer of CNN which is the Softmax layer. The output of the fully connected layer will represent a feature vector that contains five features, each feature represents one type of the class [25]. Such feature extraction methodology is fully automated, it results in very deep and efficient features, the features can extract representative features for the entered data, especially when the used $\mathrm{CNN}$ is well designed and trained on a large dataset [26]. The dimension of the extracted features used in this research is $M \times N$ where $M$ represents the number of Images, and $N$ is the number of classes (Five in our case) [25].

\subsection{Classifiers}

After extracting the deep features successfully, the classifier(s) is needed to classify these features to find the corresponding class for any test image. For this purpose; in this research; eight different classifiers rather than Softmax were used including Linear Support Vector Machine (LSVM), Radial Basis Function-SVM (RBF 
SVM), Artificial Neural Network (ANN), K Nearest Neighbor $(\mathrm{KNN})$, Random Forest (RF), Linear Discriminant Analysis (LDA), Quadratic Discriminant Analysis (QDA), and

Naïve Bayes (NB).

\subsubsection{Support Vector Machine (SVM)}

Support Vector Machine (SVM) is one of the most well-known and widely used supervised machine learning algorithms, mainly used to classify data into two classes. The SVM algorithm uses the entered training data to build a model that expects the new samples class, the model built by determining the optimal hyperplane that separates the dataset. This hyperplane must maximize the margin between the nearest data point and the separating hyperplane. The SVM was successfully applied to a wide range of real-world applications especially Biomedical Engineering, including face detection, recognition, and verification, image retrieval, handwritten character, and digit recognition [27],[28].

\subsubsection{Artificial Neural Network (ANN)}

Artificial neural network (ANN) is one of the most well-developed machine learning algorithms that replicate the actions of human brain neurons. These neurons; connected by weight-bearing linkages; form the neural model of the artificial neural networks. Different ANNs built to model specific tasks based on the role that will be performed, where various types of neural networks assume specific modes of operation for the network. There are essentially two types of feed-forward type or feedback type [29], [30].

\subsubsection{K-Nearest Neighbor (KNN)}

$\mathrm{K}$-nearest neighbors (KNN) algorithm is one of the simplest, nonparametric, lazy, and instant learning methods used in two main types of problems in machine learning, namely: classification and regression. In both instances, the input vector consists of function space, and the result is a class member that is classified based on the plurality voting technique of a neighbors class, while the object is assigned to the main class that belongs to that class. The majority vote shall refer to the weights representing the difference between each function point and the center of mass of the vector [29], [30].

\subsubsection{Random Forest (RF)}

The Random Forests (RF) Classifier was first introduced by Breiman [30] and is one of the most common and commonly used Ensemble Machine Learning Techniques. The basic principle of $\mathrm{RF}$ is to create classification trees using randomly selected features from randomly selected samples with a baggage strategy. These trees can then be used to vote for a given input vector to get a class name. RF has many benefits, such as flexibility in large-scale data, high precision, it can be effectively extended to multi-class inputs, and it does not interfere.

\subsubsection{Discriminant Analysis (DA)}

Discriminant analysis (DA) is a very popular method of classification, commonly used and statistically based, that uses training features to estimate parameters that can construct the model to distinguish and isolate the various forecasting variables from the discriminating surface functions. Discriminatory analyzes have an alternate surface for which they are linear and then called Linear Discriminant Analysis (LDA), while one is quadratic and then Quadratic Discriminant Analysis (QDA). The linear discrimination function is used to fit a normal multivariate density into any group, and the covariance matrix is determined by a sample, while the multivariate discriminant function is used to fit a normal multivariate density with sample stratification covariance estimates [31].

\subsubsection{Naïve Bayes (NB)}

Naïve Bayes (NB) classifiers are a family of basic probabilistic classifiers focused on the implementation of Bayes' theorem, with clear naive assumptions of independence between the functions. But they can be linked to an estimation of the Kernel Density and reach higher accuracy levels. They are among the simplest Bayesian network models. Naïve Bayes has been studied extensively since the early 1960 s and remains a popular method till now. NB Classifiers are extremely flexible, allowing a range of linear parameters for the number of features and predictors of learning difficulties. Maximum-likelihood testing may be achieved by analyzing a closed-form expression that requires linear time rather than an inefficient iterative approximation as used by certain other forms of classifiers [32].

\subsection{Performance Evaluation}

In any artificial intelligence (AI) based medical diagnosis system; there must be an evaluation of the system performance regarding any new data to measure its ability to determine the class of unknown causes. So; to evaluate the performance of the proposed system, the original annotations of the SD-OCT images were compared with the predicted annotations by the system from the same images. After that, using the original and precited annotations the accuracy, sensitivity, precision, and specificity are calculated, where this evaluation parameter measures how precisely; the SDOCT images are diagnosed [28], [29]. To compute these measurements, four different types of statistical values are computed, those are true positive (TP), false positive (FP), false negative (FN), and true negative (TN) [30]. Then, using these values, the mentioned measurements are computed as follows:

$$
\begin{aligned}
& \text { Accuracy }=\frac{\mathrm{TP}+\mathrm{TN}}{\mathrm{TP}+\mathrm{FP}+\mathrm{TN}+\mathrm{FN}} \\
& \text { Sensitivity }=\frac{\mathrm{TP}}{\mathrm{TP}+\mathrm{FN}} \\
& \text { Precision }=\frac{\mathrm{TP}}{\mathrm{TP}+\mathrm{FP}} \\
& \text { Specificity }=\frac{\mathrm{TN}}{\mathrm{FP}+\mathrm{TN}}
\end{aligned}
$$

\section{Results}

The proposed hybrid system was tested using a hardware environment with a desktop computer with Intel Core I7-6700 at $3.4 \mathrm{GHz}$ and $16 \mathrm{~GB}$ of RAM and the codes were executed in a parallel environment. First, the system was used for extracting the deep features from both training and testing datasets. As mentioned before the deep features are representative where only a single feature will be extracted for each class. Figure 5 shows the extracted deep features for both training and testing datasets.

After that, the extracted deep training features are used to train all five models (SVM, ANN, KNN, RF, and QDA). The SVM model was trained using two different kernels linear and radial basis function (RBF), ANN using 50 hidden layers, the number of neighbors of KNN is 1 and distance is euclidean, and RF with 100 as several bags. These models will be tested later using the testing features. Figure 6 shows the confusion matrices for all classifiers during the training phase. 

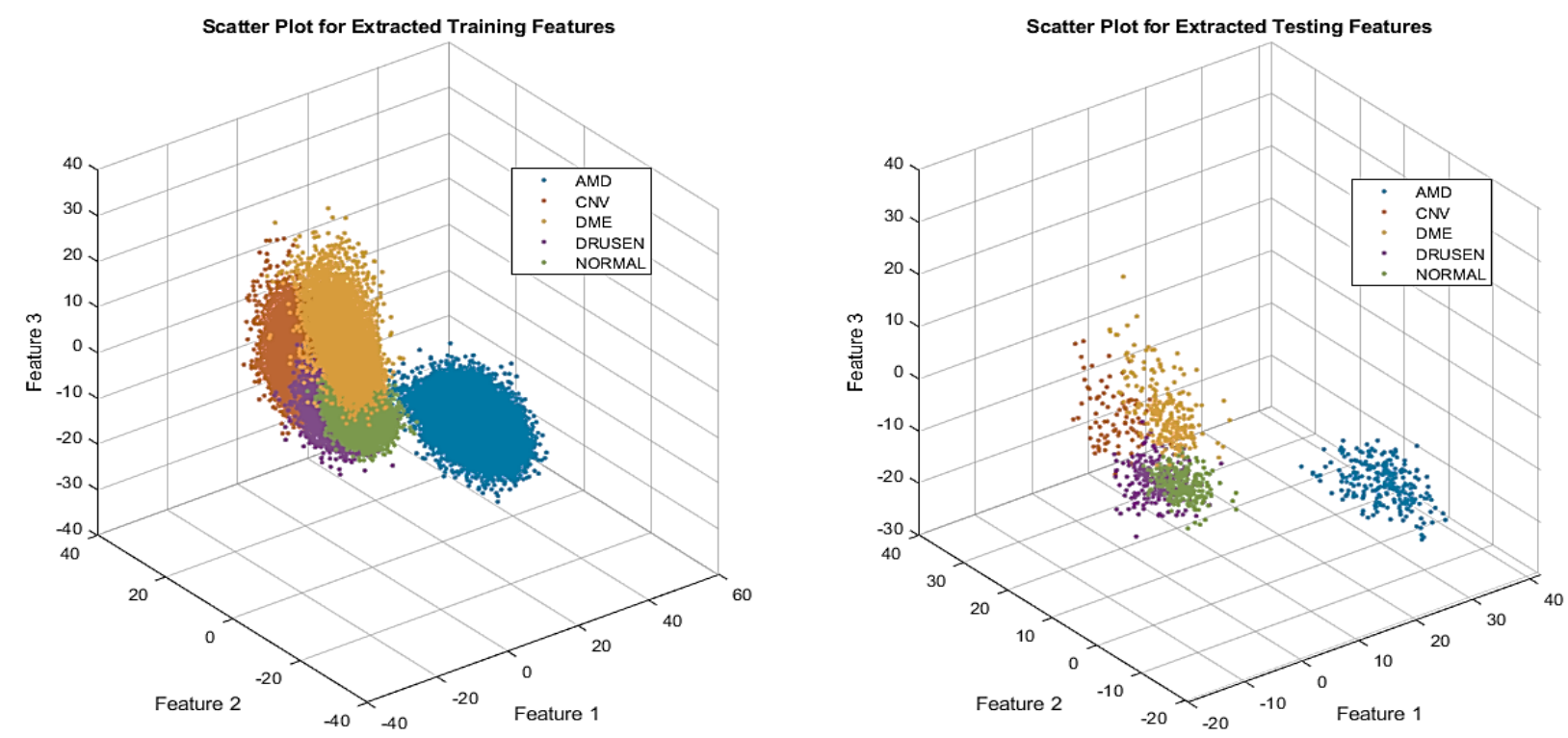

Fig. 5. Extracted Deep Features using AOCTNet CNN.

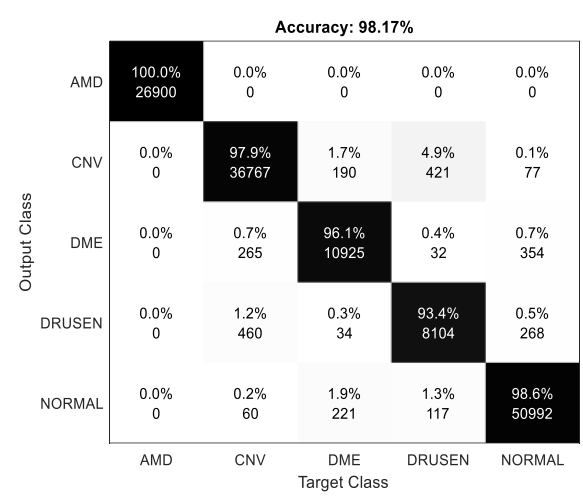

(A)

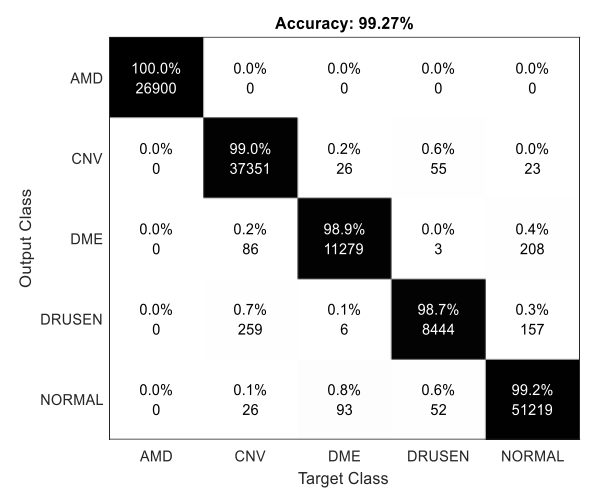

(B)

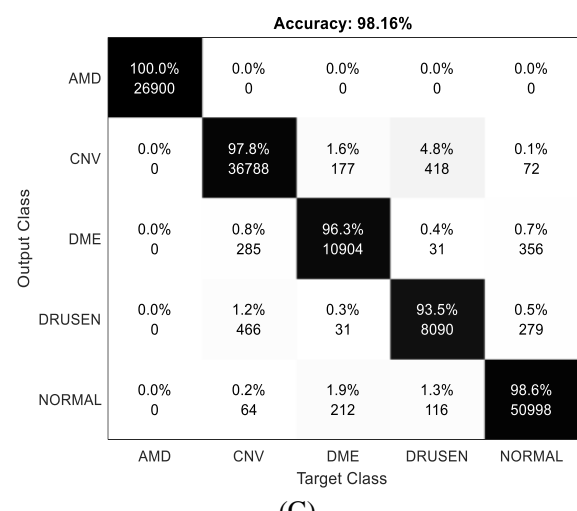

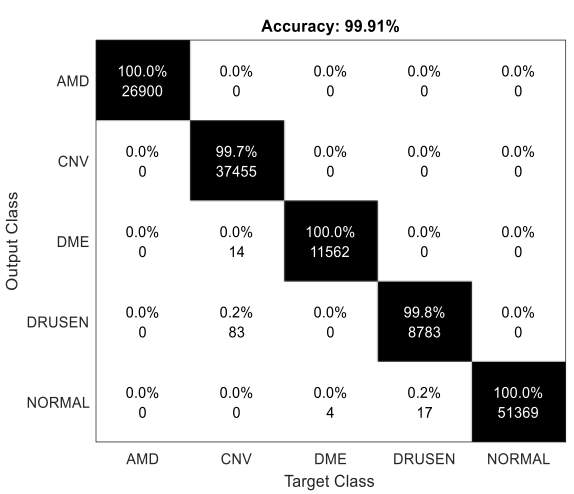

(D)

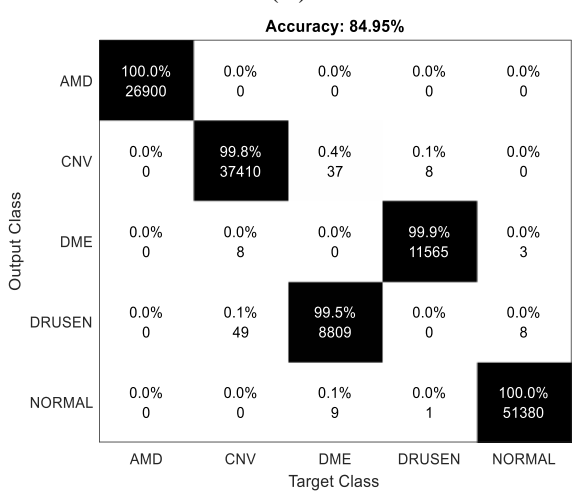

(E)

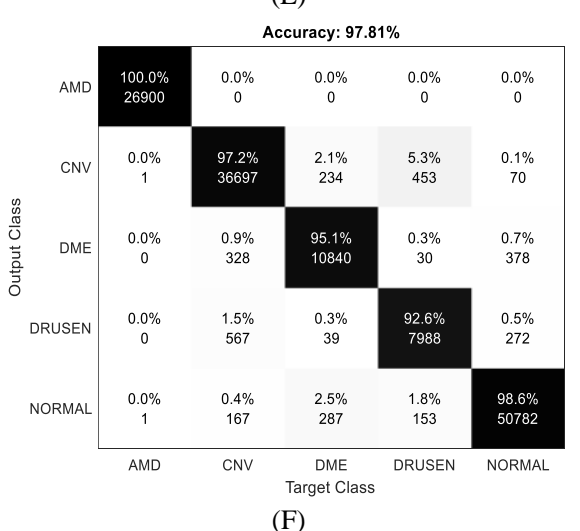




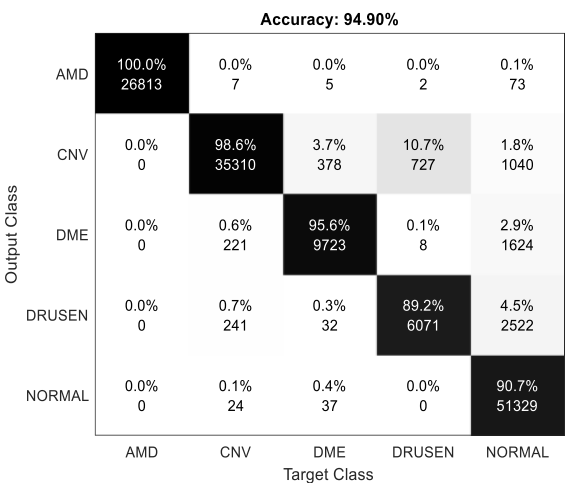

(G)

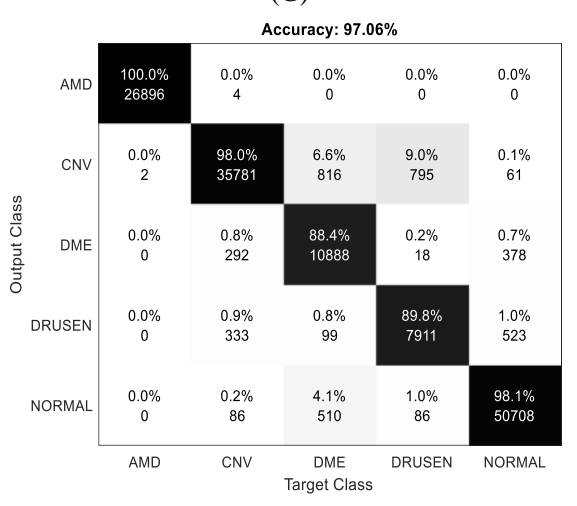

(H)

Fig. 6. Training Confusion Matrices for All used Classifiers; (A) LSVM (B) RBF SVM; (C) ANN; (D) KNN; (E) RF; (F) QDA; (G) LDA; and (H) NB

All classifiers result in high accuracy during the training phase except the random forest (RF) and Linear discriminant analysis (LDA) classifiers which are scored the two lowest training accuracies among all used classifiers. Meanwhile, all other classifiers are recoding training accuracy higher than $97 \%$ which reflects that the extracted deep features are representative and can discriminate between the eye disease classes using SD-OCT. The testing phase includes testing the stored trained models using an independent set. Then the confusion matrices for all models were plotted, Figure 7 shows the confusion matrices for all classifiers during the testing phase. Table 1 shows the detailed performance measurements of the proposed systems.

Table 1. Performance Measurements of Proposed Systems

\begin{tabular}{ccccc}
\hline \multirow{2}{*}{ Method } & Accuracy & $\begin{array}{c}\text { Mensitivity } \\
\text { \% }\end{array}$ & $\begin{array}{c}\text { Specificity } \\
\text { \% }\end{array}$ & $\begin{array}{c}\text { Precision } \\
\text { \% }\end{array}$ \\
\hline LSVM & $\mathbf{9 7 . 2 8}$ & 97.28 & 99.32 & 97.33 \\
RBF SVM & $\mathbf{9 8 . 5 6}$ & 98.56 & 99.64 & 98.57 \\
ANN & $\mathbf{9 7 . 4 4}$ & 97.44 & 99.36 & 97.48 \\
KNN & 99.44 & 99.44 & 99.86 & 99.45 \\
RF & 99.12 & 99.12 & 99.78 & 99.12 \\
QDA & 96.96 & 96.96 & 99.24 & 97.04 \\
LDA & 91.44 & 91.44 & 97.86 & 92.89 \\
NB & 97.28 & 97.28 & 99.32 & 97.36 \\
\hline
\end{tabular}

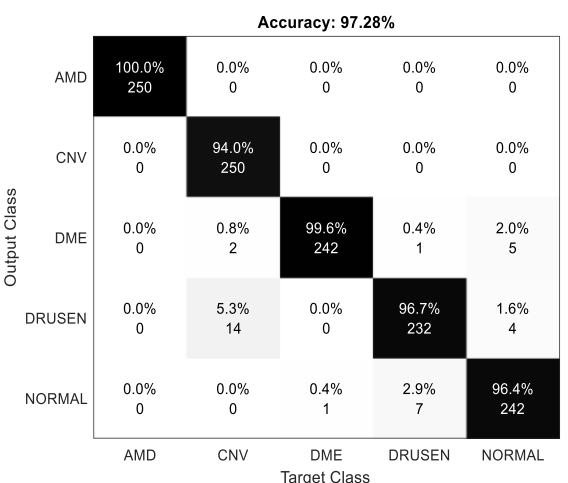

(A)

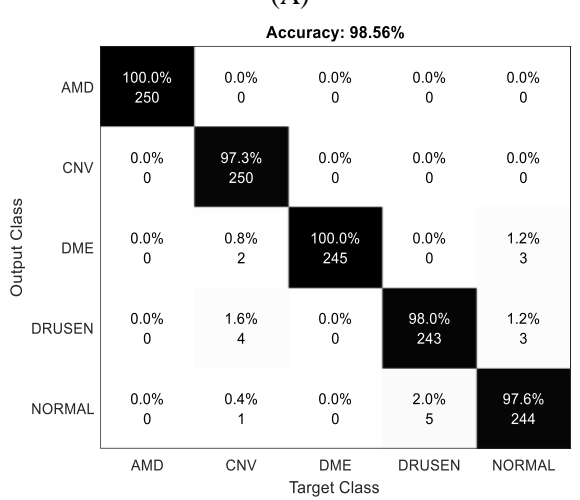

(B)

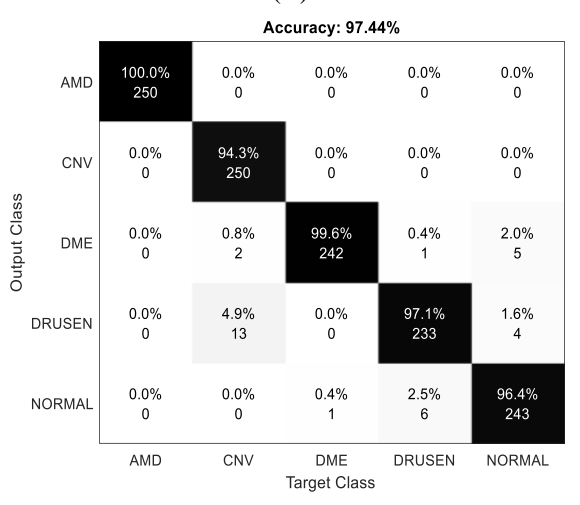

(C)

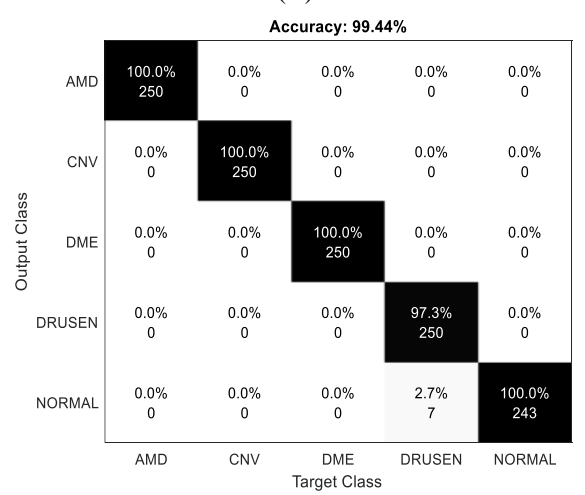

(D) 


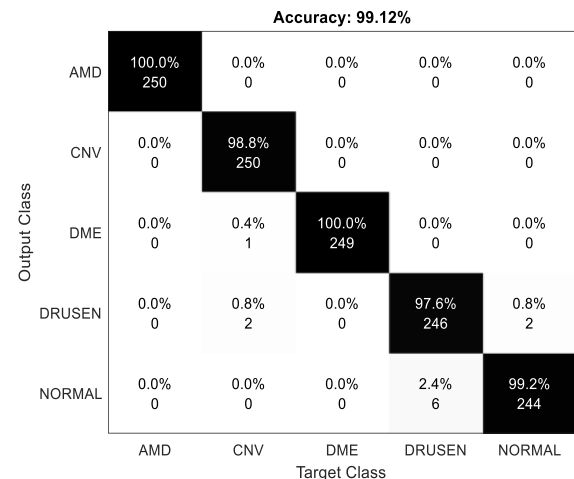

(E)

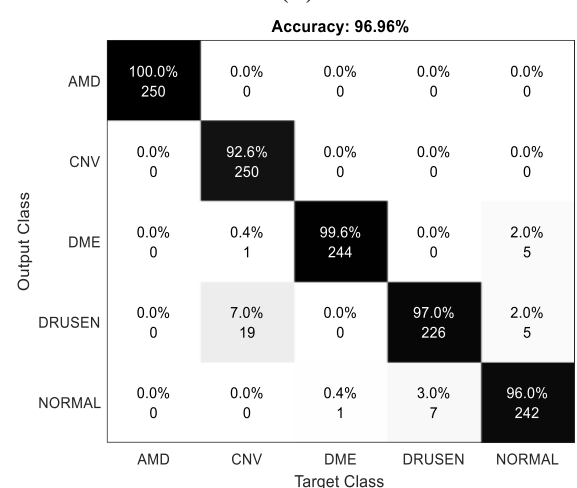

(F)

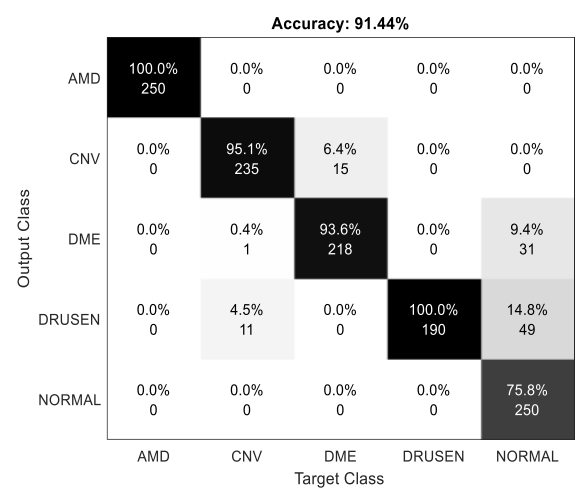

(G)

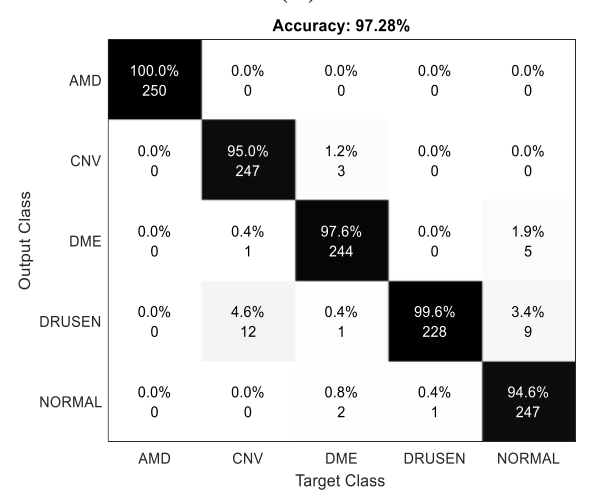

(H)

Fig. 7. Testing Confusion Matrices for All used Classifiers; (A) LSVM; (B) RBF SVM; (C) ANN; (D) KNN; (E) RF; (F) QDA; (G) LDA; and (H) NB.

\section{Discussion}

Comparing the proposed system results with other systems in the literature is presented in Table 2. All surveyed literature research listed and compared in Table 2 uses multiple SD-OCT databases or a mix of datasets to create a large one It should be noted that the majority of the research in the literature uses a different number of classes which is usually two or three classes and only four research used four classes. Such factors may affect the performance of the classification methods significantly. Also, it is noted that the researchers in surveyed literature focused on pre-processing methods and used focus in using the pre-trained CNN models rather than designing CNN models, these models are applied using transferee learning techniques to make it able to take un preprocessed images. Also, from Table 2 we can observe that different pre-trained $\mathrm{CNN}$ models were used with the transferee learning technique. These models include Inception V2, Inception V3, and GoogleNet models were used, on the other hand, some papers make ensemble methodologies using these pre-trained CNN models were proposed.

Moreover, some papers in the literature use machine learning algorithms on features extracted from the segmented region of interest (ROI) of SD-OCT. Moreover, most of the listed methods in the literature have achieved high recognition rates, more than $80 \%$. As a final note, most methods in the surveyed literature were used to make a classification of the OCT images for three diseases classes, while there are only four papers that proposed a system to classify them into four classes and only one method to classify images into five classes. Also, we used the largest dataset of SDOCT images used with 137,437 images while the closest one was $113,397$.

Finally, using Table 2 the proposed hybrid system achieves the highest accuracy for classifying the SD-OCT images with the largest used dataset of SD-OCT images that have been used in such a system, while it is the second system that is able to classify the images to five classes but with superior performance compared to other systems. The proposed hybrid systems show that such systems achieve a higher classification rate compared to other methods. While comparing the elapsed time of each classifier in classifying a new image, the average time for classifying all testing for each classifier was measured and shown in Figure 8.

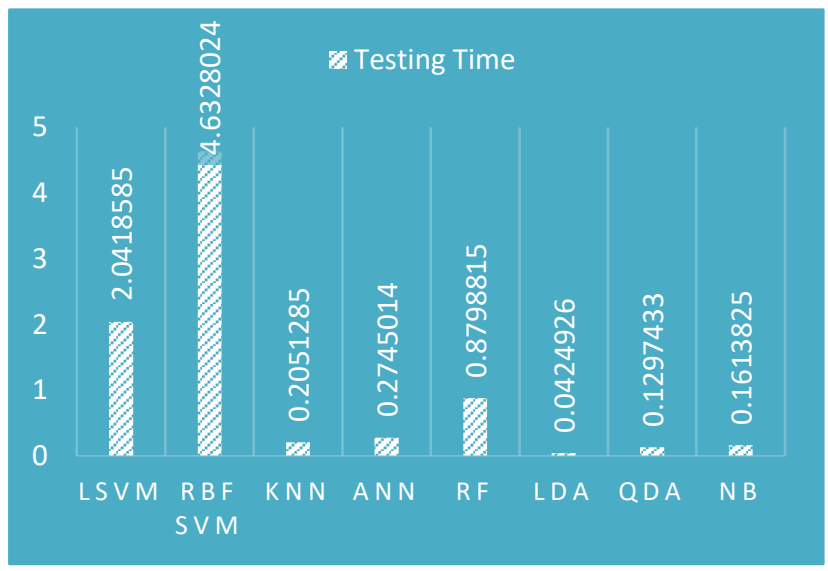

Fig. 8. Average Testing Time of all Used Classifiers in Seconds.

Using Table 1 and Figure 8 we see that the best classifier among all performance measurements and time consumption is the KNN. This means that this classifier can classify the SD-OCT images with the highest performance and the shortest time among all used classifiers.

\section{Conclusion}

Modern lifestyle diseases have made a tight grip around our daily lives. Eye retinal diseases being one of the most common diseases that occur around the world that change or affect the life of many 
people. Eye retinal disease remains a problem to be solved at its very basic level. In this paper, an artificial intelligence hybrid system is proposed for enhancing eye retinal diseases diagnosis. The hybrid systems are based using automated deep features extracted from SD-OCT images using AOCTNet, then these features are fed into eight different classifiers. Furthermore, the proposed systems achieved an accuracy better than the scoped works in the included literate, in addition to the AOCTNet itself. The experimental results show that the CNN-KNN systems achieved the highest accuracy rate of $99.44 \%$.

It is worth mentioning that the proposed system has the following advantages: First, it is considered the first artificial intelligence hybrid system for OCT classification. Second, deep features extraction has been automated. Third, the classification of the OCT images has been enhanced. Lastly, the proposed system is fast and robust. Nevertheless, the proposed system needs a long time for feature extraction.

Table 2. Comparison between the literature works and the proposed system

\begin{tabular}{|c|c|c|c|c|}
\hline Ref \# & Technique & $\begin{array}{c}\text { Dataset } \\
\text { Size }\end{array}$ & Classes & Accuracy $\%$ \\
\hline [1] & CNN & 137,437 & 5 & 97.1 \\
\hline [17] & $\begin{array}{l}\text { Transfer } \\
\text { learning with } \\
\text { GoogleNet C } \\
\text { NN }\end{array}$ & 10,000 & 3 & 96 \\
\hline [20] & $\mathrm{CNN}$ & 4,096 & 2 & 93 \\
\hline [21] & $\begin{array}{l}\text { SVM with } \\
\text { HOG Features } \\
\text { Transfer } \\
\text { learning with }\end{array}$ & 3,247 & 3 & 95.56 \\
\hline [21] & $\begin{array}{l}\text { Inception } \\
\text { Resnet V2 } \\
\text { CNN }\end{array}$ & 113,397 & 4 & 86 \\
\hline [33] & $\mathrm{RF}$ & 1,183 & 3 & 96 \\
\hline [34] & CNN & 3,231 & 2 and 3 & 91.77 \\
\hline \multirow[t]{4}{*}{ [35] } & IFCNN & 84,484 & 4 & 89.5 \\
\hline & Linear SVM & 137,437 & 5 & 97.28 \\
\hline & RBF SVM & 137,437 & 5 & 98.56 \\
\hline & KNN & 137,437 & 5 & 99.44 \\
\hline This & ANN & 137,437 & 5 & 97.44 \\
\hline \multirow[t]{4}{*}{ Paper } & $\mathrm{RF}$ & 137,437 & 5 & 99.12 \\
\hline & QDA & 137,437 & 5 & 96.96 \\
\hline & LDA & 137,437 & 5 & 91.44 \\
\hline & NB & 137,437 & 5 & 97.28 \\
\hline
\end{tabular}

\section{Abbreviations}

CNN: Convolutional Neural Network; OCT: Optical Coherence Tomography; AOCTNet: Advanced OCT Network; AMG: agerelated macular degeneration; SD-OCT: spectral-domain optical coherence tomography; CNV: choroidal neovascularization; DME: diabetic macular edema; SVM: Support Vector Machine; ANN: Artificial Neural Network; KNN: K Nearest Neighbor; RF: Random Forest; DA: Discriminant Analysis; NB: Naïve Bayes; LDA: Linear Discriminant Analysis; QDA: Quadratic Discriminant Analysis; HOG: histogram of oriented gradients; AI: artificial intelligence; ML: Machine Learning; DL: Deep Learning; FC: fully connected; TP: True positive; TN: True negative; FP: False positive; FN: False negative.

\section{Acknowledgment}

The authors would like to thank the Nvidia Learning Center at Yarmouk University (YU), represented by Dr. Ahmad M. Alomari, for providing access to the GPU Unit

\section{References}

[1] Alqudah, A. M. (2020). AOCT-NET: a convolutional network automated classification of multiclass retinal diseases using spectraldomain optical coherence tomography images. Medical \& biological engineering \& computing, 58(1), 41-53. https://doi.org/10.1007/s11 517-019-02066-y

[2] Kermany, D. S., Goldbaum, M., Cai, W., Valentim, C. C., Liang, H., Baxter, S. L., ... \& Dong, J. (2018). Identifying medical diagnoses and treatable diseases by image-based deep learning. Cell, 172(5), 11221131. https://doi.org/10.1016/j.cell.2018.02.010

[3] Farsiu, S., Chiu, S. J., O'Connell, R. V., Folgar, F. A., Yuan, E., Izatt, J. A., Toth, C.A. (2014). Quantitative classification of eyes with and without intermediate age-related macular degeneration using optical coherence tomography. Ophthalmology, 121(1), 162-172. https://d oi.org/10.1016/j.ophtha.2013.07.013

[4] Lemaître, G., Rastgoo, M., Massich, J., Cheung, C. Y., Wong, T. Y., Lamoureux, E., ... \& Sidibé, D. (2016). Classification of SD-OCT volumes using local binary patterns: experimental validation for DME detection. Journal of ophthalmology, 2016. https://doi.org/10.1155/2016/3298606

[5] Reis, A. S., O'Leary, N., Yang, H., Sharpe, G. P., Nicolela, M. T., Burgoyne, C. F., \& Chauhan, B. C. (2012). Influence of clinically invisible, but optical coherence tomography detected, optic disc margin anatomy on neuroretinal rim evaluation. Investigative ophthalmology \& visual science, 53(4), 1852-1860. https://doi.org/10.1167/iovs.11-9309

[6] Kanagasingam, Y., Bhuiyan, A., Abramoff, M. D., Smith, R. T., Goldschmidt, L., \& Wong, T. Y. (2014). Progress on retinal image analysis for age related macular degeneration. Progress in retinal and eye research, 38, 20-42. https://doi.org/10.1016/j.preteyeres .2013.10.002

[7] Esteva, A., Robicquet, A., Ramsundar, B., Kuleshov, V., DePristo, M., Chou, K., ... \& Dean, J. (2019). A guide to deep learning in healthcare. Nature medicine, 25(1),

https://doi.org/10.1038/s41591-018-0316-Z

[8] Schmidt-Erfurth, U., Sadeghipour, A., Gerendas, B. S., Waldstein, S. M., \& Bogunović, H. (2018). Artificial intelligence in retina. Progress in retinal and eye research, 67, 1-29. https://doi.org/10.1016/j.preteyeres. 2018.07.004

[9] Ting, D. S., Wu, W. C., \& Toth, C. (2019). Deep learning for retinopathy of prematurity screening. http://dx.doi.org/10.1136/bjophthalmol-2018-313290

[10]Ting, D. S. W., Pasquale, L. R., Peng, L., Campbell, J. P., Lee, A. Y., Raman, R., ... \& Wong, T. Y. (2019). Artificial intelligence and deep learning in ophthalmology. British Journal of Ophthalmology, 103(2), 167-175. http://dx.doi.org/10.1 136 /bjophthalmol-2018-313173

[11]Pierro, L., Zampedri, E., Milani, P., Gagliardi, M., Isola, V., \& Pece, A. (2012). Spectral domain OCT versus time domain OCT in the evaluation of macular features related to wet age-related macular degeneration. Clinical ophthalmology (Auckland, NZ), 6, 219. https://doi.org/10.2147/OPTH.S27656

[12] Sajda, P. (2006). Machine learning for detection and diagnosis of disease. Annu. Rev. Biomed. Eng., 8, 537565.https://doi.org/10.1146/annurev.bioeng.8.061505.095802

[13]LeCun, Y., Bengio, Y., \& Hinton, G. (2015). Deep learning. nature, 521(7553), 436-444. https://doi.org/10. 1038/nature14539

[14]Alsaih, K., Lemaitre, G., Rastgoo, M., Massich, J., Sidibé, D., \& Meriaudeau, F. (2017). Machine learning techniques for diabetic macular edema (DME) classification on SD-OCT images. Biomedical engineering online, 16(1), 68. https://doi.org/10.11 86/s12938-0170352-9 
[15] Awais, M., Müller, H., Tang, T. B., \& Meriaudeau, F. (2017, September). Classification of sd-oct images using a deep learning approach. In 2017 IEEE International Conference on Signal and Image Processing Applications (ICSIPA) (pp. 489-492). IEEE. https://doi.org/10.1109/ICSIPA.2017.8120661

[16]Lee, C. S., Baughman, D. M., \& Lee, A. Y. (2017). Deep learning is effective for classifying normal versus age-related macular degeneration OCT images. Ophthalmology Retina, 1(4), 322-327. https://doi.org/10.1016/j.oret.2016.12.009

[17]Karri, S. P. K., Chakraborty, D., \& Chatterjee, J. (2017). Transfer learning based classification of optical coherence tomography images with diabetic macular edema and dry age-related macular degeneration. Biomedical optics express, 8(2), 579-592. https://doi.org/10.1364/boe.8.000579

[18] Amil, P., González, L., Arrondo, E., Salinas, C., Guell, J. L., Masoller, C., \& Parlitz, U. (2019). Unsupervised feature extraction of anterior chamber OCT images for ordering and classification. Scientific reports, 9(1), 1-9. https://doi.org/10.1038/s41598-018-38136-8

[19] Ji, Q., He, W., Huang, J., \& Sun, Y. (2018). Efficient deep learningbased automated pathology identification in retinal optical coherence tomography images. Algorithms, 11(6), 88. https://doi.org/10.3390 /a11060088

[20]Perdomo, O., Otálora, S., González, F. A., Meriaudeau, F., \& Müller, H. (2018, April). Oct-net: A convolutional network for automatic classification of normal and diabetic macular edema using sd-oct volumes. In 2018 IEEE 15th International Symposium on Biomedical Imaging (ISBI 2018) (pp. 1423-1426). IEEE. https://doi.org/10.1109/ISBI.2018.8363839

[21]Nugroho, K. A. (2018, October). A comparison of handcrafted and deep neural network feature extraction for classifying optical coherence tomography (OCT) images. In 2018 2nd International Conference on Informatics and Computational Sciences (ICICoS) (pp. 1-6). IEEE. https://doi.org/10.1109/ICICOS.2018.8621 687

[22] Hwang, D. K., Hsu, C. C., Chang, K. J., Chao, D., Sun, C. H., Jheng, Y. C., ... \& Peng, C. H. (2019). Artificial intelligence-based decisionmaking for age-related macular degeneration. Theranostics, 9(1), 232. https://doi.org/10.7150/thno.28447

[23] Gnanadurai, D., \& Sadasivam, V. (2005). Image de-noising using double density wavelet transform based adaptive thresholding technique. International Journal of Wavelets, Multiresolution and Information Processing, 3(01), 141-152. https://doi.org/10.1142/S02 19691305000701

[24] A.M. Alqudah, H. Alquraan, I. Abu Qasmieh, A. Alqudah and W. AlSharu (2019). Brain Tumor Classification Using Deep Learning Technique - A Comparison between Cropped, Uncropped, and Segmented Lesion Images with Different Sizes, International Journal of Advanced Trends in Computer Science and Engineering, vol. 8, no. 6, pp. 3684-3691, 2019. https://doi.org/10.30534/ijatcse/20 $19 / 155862019$

[25]Trosten, D. J., \& Sharma, P. (2019, June). Unsupervised feature extraction-a cnn-based approach. In Scandinavian Conference on Image Analysis (pp. 197-208). Springer, Cham. https://doi.org/10.1007/978-3-030-20205-7_17

[26] Garcia-Gasulla, D., Parés, F., Vilalta, A., Moreno, J., Ayguadé, E., Labarta, J., .. \& Suzumura, T. (2018). On the behavior of convolutional nets for feature extraction. Journal of Artificial Intelligence Research, 61, 563-592. https://doi.org/10.1613/jair.5756
[27] Alqudah, A. M., Algharib, H. M., Algharib, A. M., \& Algharib, H. M. (2019). Computer aided diagnosis system for automatic two stages classification of breast mass in digital mammogram images. Biomedical Engineering: Applications, Basis and Communications, 31(01), $1950007 . \quad$ https://doi.org/10.40 15/S1016237219500078

[28] Alqudah, A., \& Alqudah, A. M. (2019). Sliding window based support vector machine system for classification of breast cancer using histopathological microscopic images. IETE Journal of Research, 1-9. https://doi.org/10.1080/03772063.2019.1583610

[29]Alqudah, A. M. (2019). Ovarian Cancer Classification Using Serum Proteomic Profiling and Wavelet Features A Comparison of Machine Learning and Features Selection Algorithms. Journal of Clinical Engineering, 44(4), 165-173. https://doi.org/10.1097/JC E.0000000000000359

[30] Alqudah, A. M. (2019). Towards classifying non-segmented heart sound records using instantaneous frequency based features. Journal of medical engineering \& technology, 43(7), 418-430. https://doi.org/10.1080/ 03091902.2019.1688408

[31]Alkan, A., \& Günay, M. (2012). Identification of EMG signals using discriminant analysis and SVM classifier. Expert systems with Applications, 39(1),

$44-47$ https://doi.org/10.1016/j.eswa.2011.06.043

[32]Dey Sarkar, S., Goswami, S., Agarwal, A., \& Aktar, J. (2014). A novel feature selection technique for text classification using Naive Bayes. International scholarly research notices, 2014 https://doi.org/10.1155/2014/ 717092

[33] Hussain, M. A., Bhuiyan, A., Luu, C. D., Smith, R. T., Guymer, R. H., Ishikawa, H., ... \& Ramamohanarao, K. (2018). Classification of healthy and diseased retina using SD-OCT imaging and Random Forest algorithm. PloS one, 13(6). https://doi.org/10.1371/jou rnal.pone.0198281

[34]Rong, Y., Xiang, D., Zhu, W., Yu, K., Shi, F., Fan, Z., \& Chen, X. (2018). Surrogate-assisted retinal OCT image classification based on convolutional neural networks. IEEE journal of biomedical and health informatics, 23(1), 253-263. https://doi.org/10.1109/JB HI.2018.2795545

[35]Fang, L., Jin, Y., Huang, L., Guo, S., Zhao, G., \& Chen, X. (2019). Iterative fusion convolutional neural networks for classification of optical coherence tomography images. Journal of Visual Communication and Image Representation, 59, 327-333. https://doi.org/10.1016/j.j vcir.2019.01.022.

[36]Luo Y, Pan J, Fan S, Du Z, Zhang G. Retinal image classification by self-supervised fuzzy clustering network. IEEE Access. 2020 May 12;8:92352-62.

[37]He, X., Deng, Y., Fang, L. and Peng, Q., 2021. Multi-Modal Retinal Image Classification With Modality-Specific Attention Network. IEEE Transactions on Medical Imaging, 40(6), pp.1591-1602.

[38] Miere, A., Le Meur, T., Bitton, K., Pallone, C., Semoun, O., Capuano, V., Colantuono, D., Taibouni, K., Chenoune, Y., Astroz, P. and Berlemont, S., 2020. Deep learning-based classification of inherited retinal diseases using fundus autofluorescence. Journal of Clinical Medicine, 9(10), p.3303. 